\title{
Quercetin inhibits angiogenesis through thrombospondin-1 upregulation to antagonize human prostate cancer PC-3 cell growth in vitro and in vivo
}

\author{
FEIYA YANG ${ }^{1}$, XIAN JIANG ${ }^{2}$, LIMING SONG $^{1}$, HUIPING WANG $^{3}$, ZHU MEI $^{4}$, \\ ZHIQING $\mathrm{XU}^{4}$ and NIANZENG XING ${ }^{1}$ \\ ${ }^{1}$ Department of Urology, Beijing Chaoyang Hospital, Capital Medical University, Beijing; ${ }^{2}$ Department of Urology, \\ The Third Hospital of Nanchang, Jiangxi; ${ }^{3}$ Department of Reproductive Immunology and Pharmacology, \\ National Research Institute for Family Planning; ${ }^{4}$ Institute of Neuroscience, Beijing Key Laboratory \\ of Neural Regeneration and Repair, Capital Medical University, Beijing, P.R. China
}

Received September 17, 2015; Accepted October 24, 2015

DOI: $10.3892 /$ or.2015.4481

\begin{abstract}
The rapid growth, morbidity and mortality of prostate cancer, and the lack of effective treatment have attracted great interests of researchers to find novel cancer therapies aiming to inhibit angiogenesis and tumor growth. Quercetin is a flavonoid compound that widely exists in the nature. Our previous study preliminarily demonstrated that quercetin effectively inhibited human prostate cancer cell xenograft tumor growth by inhibiting angiogenesis. Thrombospondin-1 (TSP-1) is the first reported endogenous anti-angiogenic factor that can inhibit angiogenesis and tumorigenesis. However, the relationship between quercetin inhibiting angiogenesis and TSP-1 upregulation in prostate cancer has not been determined. Thus, we explored the important role of TSP-1 upregulation in reducing angiogenesis and anti-prostate cancer effect of quercetin both in vitro and in vivo for the first time. After the selected doses were used for a certain time, quercetin i) significantly inhibited PC-3 and human umbilical vein endothelial cells (HUVECs) proliferation, migration and invasion in a dose-dependent manner; ii) effectively inhibited prostate cancer PC-3 cell xenograft tumor growth by $37.5 \%$ with $75 \mathrm{mg} / \mathrm{kg}$ as compared to vehicle control group, more effective than $25(22.85 \%)$ and $50 \mathrm{mg} / \mathrm{kg}(29.6 \%)$; iii) was well tolerated by BALB/c mice and no obvious toxic reactions were observed; iv) greatly reduced angiogenesis and led to higher TSP-1 protein and mRNA expression both in vitro and in vivo in a dose-dependent manner. Therefore, quercetin could increase TSP-1 expression to inhibit angiogenesis resulting in
\end{abstract}

Correspondence to: Professor Nianzeng Xing, Department of Urology, Beijing Chaoyang Hospital, Capital Medical University, 8 Gongren Tiyuchang Nanlu, Beijing 100020, P.R. China

E-mail: xingnianzeng@126.com

Key words: quercetin, prostate cancer, angiogenesis inhibition, thrombospondin-1 upregulation, $\mathrm{PC}-3$, in vitro, in vivo antagonizing prostate cancer PC-3 cell and xenograft tumor growth. The present study can lay a good basis for the subsequent concrete mechanism study and raise the possibility of applying quercetin to clinical for human prostate cancer in the near future.

\section{Introduction}

Prostate cancer occupies the first place of new cases and the second leading cause of cancer mortality in males, with an estimated 220,800 new cases and 27,540 deaths in US in 2015 (1). When prostate cancer is at the early stage, it can be cured by radical prostatectomy or radiation. However, local recurrence and distant metastasis will occur in most of these patients later (2). Androgen ablation can act as an important effective treatment for the first 1-3 years, after this period it gradually develops into castration-resistant prostate cancer (CRPC) with the characteristics of elevated PSA level, higher metastasis rate and more aggressiveness (3). At present, the widely used first-line systemic chemotherapy for CRPC is the combination of docetaxel and prednisone, but it is not a curative scheme and only prolongs overall survival time for a short period. In addition, it is associated with severe side-effects (4). Therefore, the lack of effective treatment for prostate cancer arouses a great necessity to develop novel drug therapy to overcome these shortcomings and work effectively for CRPC.

In recent years, novel cancer therapies aiming to inhibit angiogenesis and tumor growth have attracted great interests of researchers among which quercetin $\left(3,3^{\prime}, 4^{\prime}, 5,7\right.$-pentahydroxyflavone) is one of the most important naturally-occurring flavonoid compounds (5-7). A large amount of quercetin exists in vegetables and fruits particularly in onions, red wine, tea and apples, and it has demonstrated fine anti-prostate cancer property through many different mechanisms (7). Our previous study reported preliminary results that quercetin could effectively inhibit human prostate cancer cell xenograft tumor growth by inhibiting angiogenesis (8). Thrombospondin-1 (TSP-1) is a $450 \mathrm{kDa}$ extracellular calcium binding glycopro- 
tein belonging to family of thrombospondins. It is the first reported endogenous anti-angiogenic factor and the widely accepted inhibitor of angiogenesis and tumorigenesis (9). However, the relationship between quercetin inhibiting angiogenesis and TSP-1 upregulation in prostate cancer has not been reported (7). Herein, we explored the important role of TSP-1 upregulation in the angiogenesis inhibition and anti-prostate cancer effect of quercetin both in vitro and in vivo for the first time.

\section{Materials and methods}

Ethics statement. All the animal experiment procedures were approved by the Committee of Animal Experimentation and the Ethics Committee of Capital Medical University (Permit No. 2013-X-83). All the operations related with animals were in strict accordance with the requirements and guidelines of experimental animal ministry.

Chemical reagents. Quercetin, dimethyl sulfoxide (DMSO), 3-(4,5-dimethylthiazol-2-yl)-2,5-diphenyltetrazolium bromide (MTT) and hydroxypropyl- $\beta$-cyclodextrin (HP $\beta C D)$ were purchased from Sigma (St. Louis, MO, USA). RPMI-1640 medium, $0.25 \%$ trypsin-EDTA, phosphate-buffered saline (PBS) and fetal bovine serum (FBS) were purchased from HyClone (Logan, UT, USA). VascuLife VEGF medium complete kit, $0.05 \%$ trypsin-EDTA and trypsin neutralizing solution were purchased from Cascade Biologics (Invitrogen, USA). Matrigel was from BD Biosciences (Franklin Lakes, NJ, USA).

Cell lines and cell culture. Human prostate cancer androgenindependent PC-3 cells were obtained from Peking Union Medical College and cultured in RPMI-1640 medium supplemented with $10 \%$ FBS. Human umbilical vein endothelial cells (HUVECs) were purchased from Peking Union Medical College and grown in VascuLife VEGF medium. PC-3 cells and HUVECs were maintained at $37^{\circ} \mathrm{C}$ in the incubator with $95 \%$ air and $5 \% \mathrm{CO}_{2}$.

Cell culture and drug treatment. Quercetin was dissolved in DMSO to firstly form a concentration of $10 \mathrm{mM}$. It was then diluted using cell culture medium to reach the required concentrations $(25,50$ and $100 \mu \mathrm{M})$ and equal volume of variable concentrations was added to the media. Following culture for the designated time, cell proliferation rate was determined by MTT assay. Comparable amount of DMSO without quercetin acted as the vehicle control during the research process.

Cell proliferation assay. MTT assay was generally used to evaluate the cell viability in the culture after drug treatment (10). PC-3 cells at a density of $3 \times 10^{4}$ cells $/ \mathrm{ml}$ and HUVECs at a density of $5 \times 10^{4}$ cells $/ \mathrm{ml}$ were seeded into 96 -well plates in triplicate. After cultured for $24 \mathrm{~h}$ allowing both PC-3 cells and HUVECs to attach, cells were treated with variable concentrations of quercetin $(25,50$ and $100 \mu \mathrm{M})$ or DMSO for further 24, 48 and $72 \mathrm{~h}$. Four hours before the termination of each time points, $20 \mu 1$ MTT with the concentration of $5 \mathrm{mg} / \mathrm{ml}$ was added to each well and co-cultured with PC-3 cells and HUVECs for another $4 \mathrm{~h}$ in the humidified environment containing $5 \% \mathrm{CO}_{2}$ at $37^{\circ} \mathrm{C}$. Then culture medium was carefully removed leaving the formazan crystals preserved completely, which was dissolved with $150 \mu 1$ DMSO by vibration for $10 \mathrm{~min}$. The absorbance value at $570 \mathrm{~nm}$ was determined using ELISA reader. Cell proliferation inhibition rates in the three quercetin treated groups at designated time were calculated based on the following formula: Proliferation inhibition rate $=\left[1-\left(\mathrm{A}_{570}\right.\right.$ treated $/ \mathrm{A}_{570}$ control $\left.)\right] \times 100 \%(10)$.

Transwell migration assay. Transwell assay (BD Biosciences) was employed to analyze the cell migration capability. Cells $\left(1 \times 10^{4}\right)$ in serum-free medium with different concentrations of quercetin or DMSO were seeded in the upper chamber of a 24-well Transwell cluster plates in triplicate. Cell culture medium containing $10 \%$ FBS was added into the lower chamber that was separated from the upper one by an $8-\mu \mathrm{m}$ pore size filter membrane. After incubated in a $5 \% \mathrm{CO}_{2}$ incubator at $37^{\circ} \mathrm{C}$ for $24 \mathrm{~h}$, cells in the the upper surface of the filter were discarded, and the migrated cells on the lower surface were fixed in ethanol and stained with crystal violet. Finally, migrated cells were photographed with inverted microscope (magnification, $\mathrm{x} 40$ ) and counted from 5 random high powered fields (magnification, $\mathrm{x} 200$ ). The data are normalized to control as means \pm SD.

Transwell invasion assay. Transwell assay was also performed to analyze the cell invasion capability. The Matrigel $(1 \mathrm{mg} / \mathrm{ml})$ (BD Biosciences) was well-distributed inside the upper surface of Transwell filter membrane overnight at $4^{\circ} \mathrm{C}$. After it was well formed and washed two times with medium before use, cells $\left(1 \times 10^{4}\right)$ in serum-free medium with different concentrations of quercetin or DMSO were seeded in the upper compartment of the filter insert that was already coated with Matrigel. The remaining procedures were carried out as described in the above migration assay.

Capillary-like tube formation assay. Capillary-like tube formation assay was used to evaluate the inhibitory effect of quercetin on angiogenesis in vitro. Experimental procedure was performed as previously described (10). Briefly, $100 \mu 1$ Matrigel solution was added to the 96-well plates and placed in the incubator with controlled conditions to form a Matrigel gel. HUVECs $\left(1 \times 10^{4} /\right.$ well) were seeded in the Matrigel layer and cultured in VascuLife VEGF medium with quercetin $(25,50$ and $100 \mu \mathrm{M})$ or DMSO for $24 \mathrm{~h}$ at $37^{\circ} \mathrm{C}$ in $5 \% \mathrm{CO}_{2}$ environment. Tube formation was detected under microscopy and photographed (magnification, x40). The number was calculated from 5 random fields and inhibition rate was determined according to the formula: Tube formation inhibition rate $=\left[1-\left(\right.\right.$ Tubes $_{\text {treated }} /$ tubes $\left.\left._{\text {control }}\right)\right] \times 100 \%$.

Animal study. Male BALB/c nude mice 4-6 weeks old were provided by the experimental animal ministry of Capital Medical University and raised in pathogen-free environment with controlled constant 12-h light and 12-h dark cycle, suitable humidity and temperature. Mice were allowed one week to get accustomed to the new environment before the experiments.

PC-3 cells $\sim 5 \times 10^{5}$ were suspended in $100 \mu \mathrm{l}$ PBS and inoculated subcutaneously into the right back. When the 
xenograft tumor volume grew to $\sim 100 \mathrm{~mm}^{3}$, mice were randomly assigned to vehicle control and three quercetin treated groups ( $\mathrm{n}=8$ each group) and treated intraperitoneally. The therapeutic dose and regimen, administration manner and dissolution method were determined according to our preliminary experiments, our previous research results and numerous other research studies $(8,11-15)$. The concrete implementation was as follows: i) vehicle control group, vehicle of quercetin, namely $25 \% \mathrm{HP} \beta \mathrm{CD}(\mathrm{w} / \mathrm{v}$ in $\mathrm{ddH}_{2} \mathrm{O}$ ) on day 1 ; ii) quercetin treated groups, quercetin 25 , 50 and $75 \mathrm{mg} / \mathrm{kg}$ dissolved in 25\% HP $\beta \mathrm{CD}\left(\mathrm{w} / \mathrm{v}\right.$ in $\left.\mathrm{ddH}_{2} \mathrm{O}\right)$ were injected in the three separate groups on day $1(8,16)$; iii) three days was a treatment cycle during which mice were weighed and xenograft tumors were measured by slide caliper, and the treatment process was terminated 4 weeks later. Tumor volume was obtained on the basis of the formula: $\mathrm{L} \times \mathrm{S}^{2} \times 0.5$, where $\mathrm{L}$ was the longest diameter and $\mathrm{S}$ was the shortest diameter of xenograft tumor $(8,4)$. Mental state, food and water intake, health condition and mortality rate were recorded as well (5). At the end of the experiments the mice were sacrificed by cervical dislocation following anesthesia by chloral hydrate. Xenograft tumors were taken out promptly, measured, weighed and photographed. Except for the portion preserved for immunohistochemical examination being kept in $10 \%$ neutral buffered formalin, the remaining was rapidly placed into liquid nitrogen for other biomarker analysis.

Western blot analysis. Western blot experiments were performed as previously described $(8,11)$. Briefly, after HUVECs, PC-3 cells and mice were treated with different concentrations of quercetin, proteins from cells and xenograft tumor tissues were extracted and quantified. Equivalent amount $(80 \mu \mathrm{g})$ of proteins were loaded, separated by $6-10 \%$ SDS-PAGE gels and transferred to polyvinylidene fluoride (PVDF) membrane (Pall, New York, NY, USA) which was blocked by $5 \%$ non-fat milk, incubated by primary antibody TSP-1 (1:500, Abcam) overnight at $4^{\circ} \mathrm{C}$ and GAPDH $(1: 10,000$; Sigma) for $1 \mathrm{~h}$ at room temperature, and incubated with horseradish peroxidase-conjugated secondary antibodies $(1: 2,000$; Cell Signaling) for another $1 \mathrm{~h}$ at room temperature in turn. Enhanced chemiluminescence kit (ECL Plus; Amersham Pharmacia Biotech, Piscataway, NJ, USA) was used to detect the antigen-antibody complex. GAPDH was used as the loading control.

Reverse transcription-quantitative real-time polymerase chain reaction ( $R T-q P C R)$. RT-qPCR was performed as previously described (8). Briefly, Total RNA of HUVECs, PC-3 cells and xenograft tumor tissues were isolated using TRIzol reagent (Invitrogen). The first-strand cDNA was synthesized with oligo(dT) primers by Transcriptor First Strand cDNA Synthesis Kit (Roche, Germany). Sequences of the PCR primers were as follows: TSP-1 forward, 5'-CACGCTGCAG GACAGCAT-3' and reverse, 5'-GGCCGCCTCAGCTCATT-3'. $\beta$-actin forward, 5'-CGGGAAATCGTGCGTGAC-3' and reverse, 5'-GTGGCCATCTCTTGCTCGAA-3'. SYBR-Green PCR master mix was used to quantify the cDNA under certain controlled conditions (ViiA 7 Real-Time PCR System) (both from Applied Biosystems, Foster City, CA, USA) and the samples were in triplicate. TSP-1 mRNA level was calculated based on the $2^{-\Delta \Delta \mathrm{Ct}}$ method and then normalized to $\beta$-actin expression.

Immunohistochemistry. The procedures for immunohistochemistry were carried out as previously described (8). Briefly, paraffin was removed and endogenous peroxidase activity was eliminated. Following antigen retrieval, sections were incubated with primary antibody CD31 (1:50) and CD34 (1:100) (both from Abcam) for $24 \mathrm{~h}$ at $4{ }^{\circ} \mathrm{C}$. The slides were then interacted with horseradish peroxidase-conjugated secondary antibody (1:100; Cell Signaling) at room temperature for another $30 \mathrm{~min}$. Diaminobenzidine reaction was employed to visualize the signals which were counterstained with hematoxylin. CD31- and CD34-positive vessels were counted from three random high power fields in each slide. In addition, at least three sections were analyzed independently in each group.

Statistical analysis. All the results are represented as means \pm SD. Statistical analysis and plots were performed by SPSS 17.0 and SigmaPlot 10.0, respectively. Differences between treated groups and vehicle control were compared using independent-samples t-test. P-value $<0.05$ denotes significant difference.

\section{Results}

Effect of quercetin on HUVEC and PC-3 cell proliferation. After treatment with varied doses of quercetin for the certain time, proliferation of HUVECs and PC-3 cells were inhibited in a dose- and time-dependent manner. Following culture with quercetin at 25,50 and $100 \mu \mathrm{M}$ for $24 \mathrm{~h}$, the inhibition rate was 9.2, 13.6 and $18.8 \%$, respectively, for HUVECs, and 13.2, 15.57 and $25.2 \%$, respectively, for PC-3 cells. When treated with above doses for $48 \mathrm{~h}$, HUVECs were inhibited by 12.02 , 19.91 and $31.22 \%$, respectively, and PC-3 cells were inhibited by $14.5,22.8$ and $39.3 \%$, respectively. When exposed to the three doses for $72 \mathrm{~h}$, the inhibition rate was $16.74,31.28$ and $55.36 \%$, respectively, for HUVECs, and 21.5, 32.5 and 50.9\%, respectively, for PC-3 cells (Fig. 1).

Effect of quercetin on HUVEC migration, invasion and tube formation. Transwell migration, invasion and tube formation of HUVECs were performed to evaluate the inhibitory effect of quercetin on angiogenesis in vitro. After treated with quercetin at 25,50 and $100 \mu \mathrm{M}$ for $24 \mathrm{~h}$, the migration, invasion and tube formation were also inhibited and the level was in proportion to the concentration increase. Migration inhibition rate was 13,25 and $55 \%$, invasion inhibition rate was 21,36 and $61 \%$ (Fig. 2), and tube formation inhibition rate was 11, 29 and $51 \%$, respectively (Fig. 4).

Quercetin upregulates TSP-1 protein and $m R N A$ expression in HUVECs. Effect of quercetin on TSP-1 protein and mRNA expression in HUVECs was investigated by western blotting and Q-PCR. Fig. 5A and C, show that quercetin $25 \mu \mathrm{M}$ greatly increased TSP-1 protein expression compared with vehicle control, and this phenomenon was more obvious when drug dose was increased. As for 
A

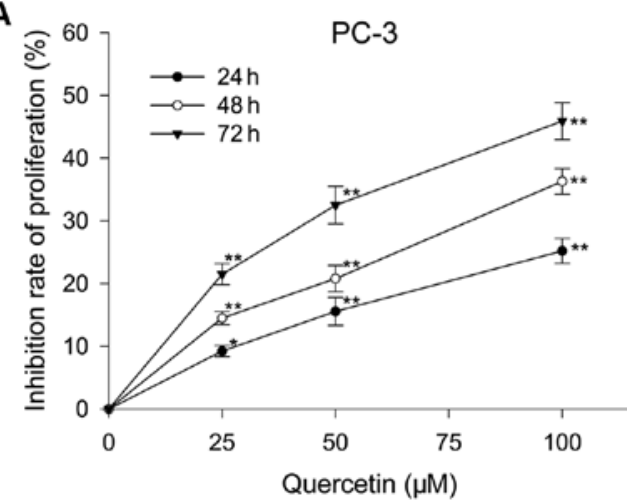

B

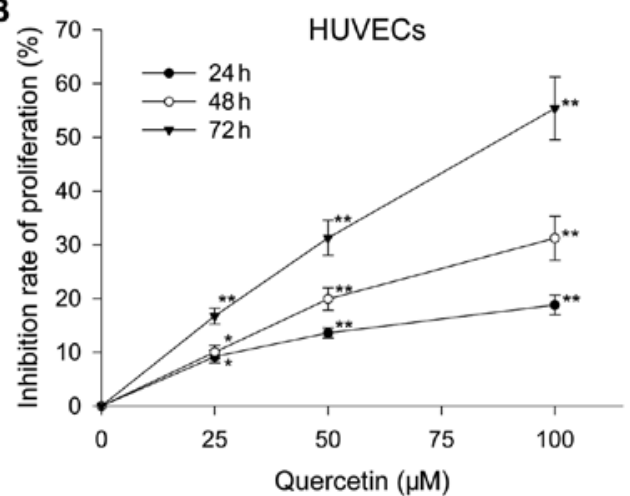

Figure 1. Quercetin inhibits human prostate cancer PC-3 cell and HUVECs growth. (A) MTT experiment showed that PC-3 cell growth was inhibited by quercetin in a dose- and time-dependent manner. (B) Quercetin inhibited HUVECs growth in a dose- and time-dependent manner. Data are presented as means $\pm \mathrm{SD}$ (mean in triplicate). ${ }^{*} \mathrm{P}<0.05$ compared with control, ${ }^{* *} \mathrm{P}<0.01$ denotes a significant difference between treatment group and the vehicle control.

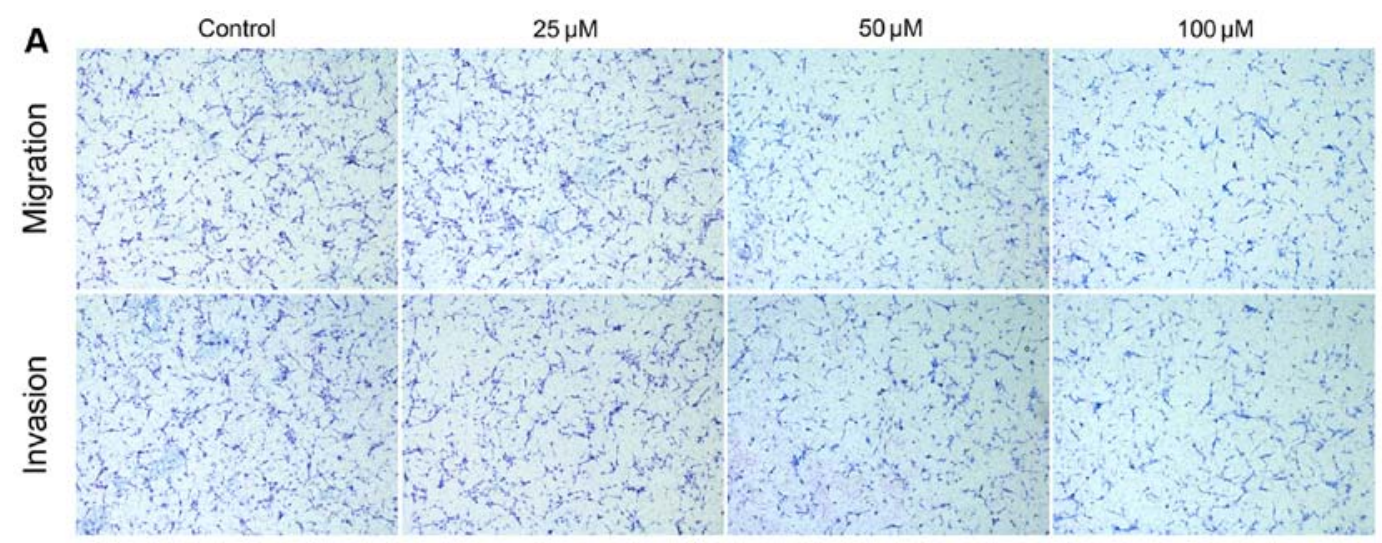

B

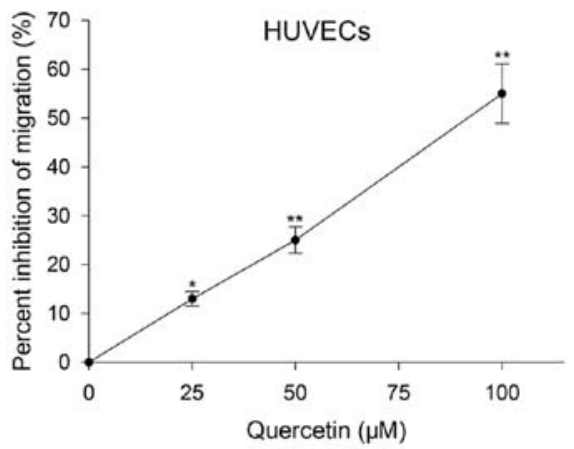

C

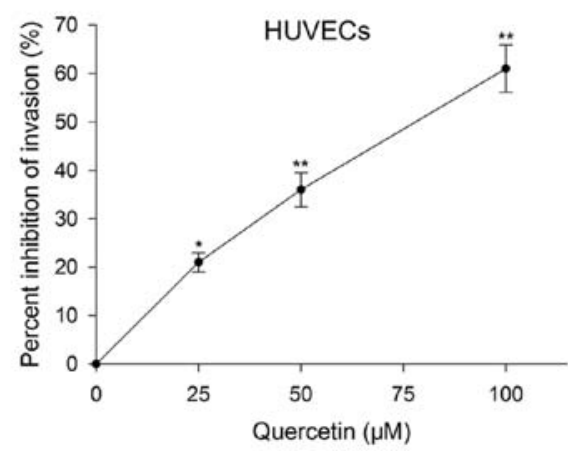

Figure 2. Quercetin inhibits migration and invasion of HUVECs in vitro. (A) Quercetin greatly inhibited both migration and invasion of HUVECs compared with vehicle control, and the inhibition rate increased when larger drug dose was used. (B and C) Inhibition rates of migration and invasion are presented as means $\pm \mathrm{SD}$ (mean in triplicate). ${ }^{*} \mathrm{P}<0.05$ compared with control, ${ }^{* *} \mathrm{P}<0.01$ denotes a significant difference between treatment and the control group.

TSP-1 mRNA (Fig. 5E), it showed the same pattern as protein with larger dose of quercetin resulting in more mRNA expression than the lower dose.

Effect of quercetin on PC-3 cell migration and invasion. Inhibitory effect of quercetin on PC-3 cells was assessed by Transwell migration and invasion. After PC-3 cells were cultured with quercetin at 25, 50 and $100 \mu \mathrm{M}$ for $24 \mathrm{~h}$, the migration inhibition rate was 17,32 and $57 \%$, and invasion inhibition was 24,36 and $69 \%$, respectively. The inhibitory degree had a positive association with the dose increase as well (Fig. 3).
Quercetin upregulates TSP-1 protein and $m R N A$ expression in PC-3 cells. TSP-1 protein and mRNA expression were detected to explore the underlying mechanisms of quercetin inhibiting PC-3 cell growth. After PC-3 cells were treated with quercetin for $24 \mathrm{~h}, 25 \mu \mathrm{M}$ greatly increased TSP-1 protein and mRNA expression compared with vehicle control group. When quercetin reached 50 and $100 \mu \mathrm{M}$, TSP-1 protein and mRNA expression was more than that of the lower dose (Fig. 5A, B and D).

Quercetin inhibits human prostate cancer $P C-3$ cell xenograft tumor growth. When PC-3 cell xenografts reached $\sim 100 \mathrm{~mm}^{3}$, 

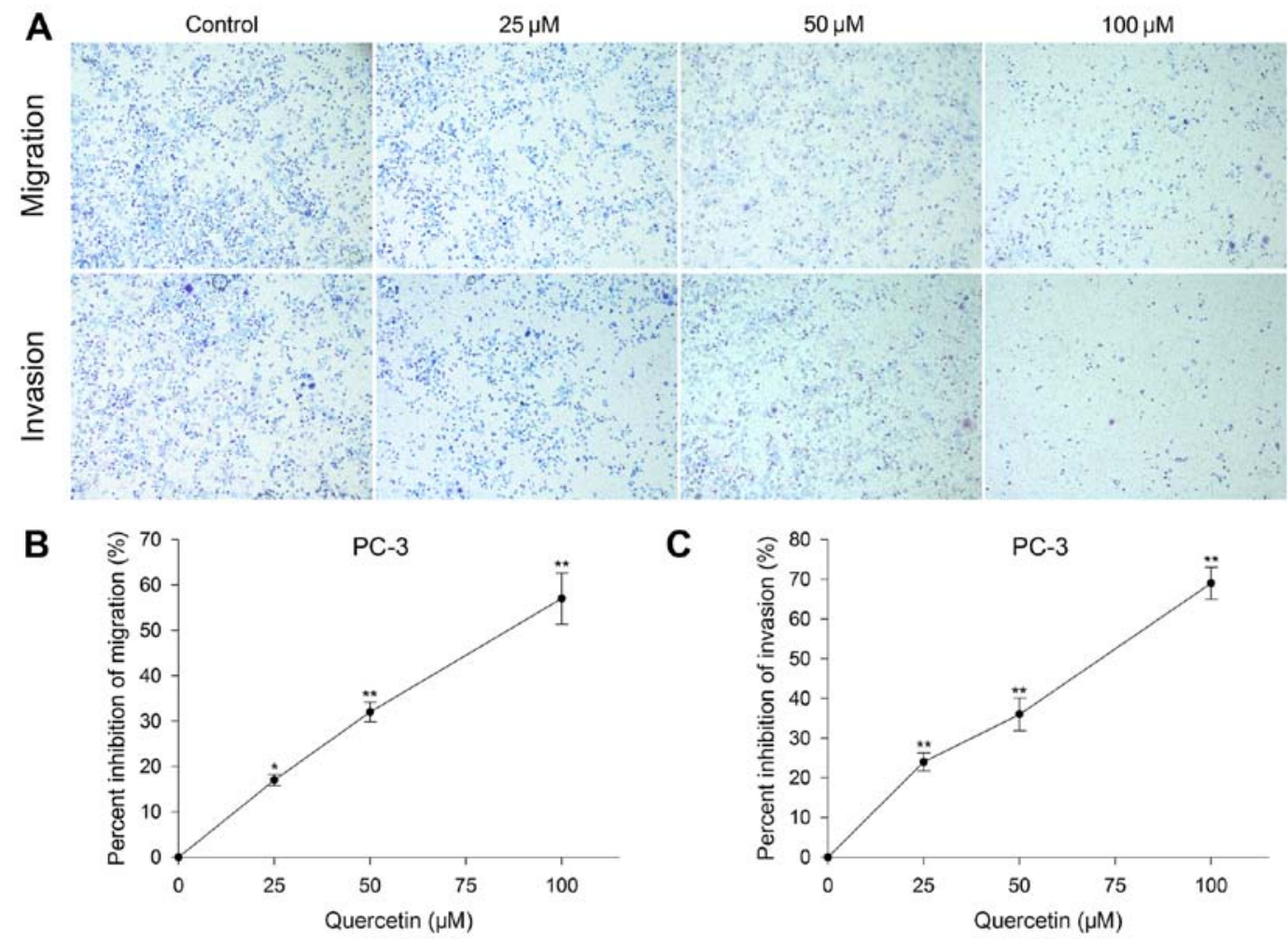

Figure 3. Quercetin inhibits migration and invasion of human prostate cancer PC-3 cells in vitro. (A) Quercetin significantly inhibited both migration and invasion of PC-3 cells in a dose-dependent manner. (B and C) Inhibition rates of migration and invasion are presented as means \pm SD (mean in triplicate). ${ }^{*} \mathrm{P}<0.05$ compared with control, ${ }^{* *} \mathrm{P}<0.01$ denotes a significant difference between treatment and the control group.
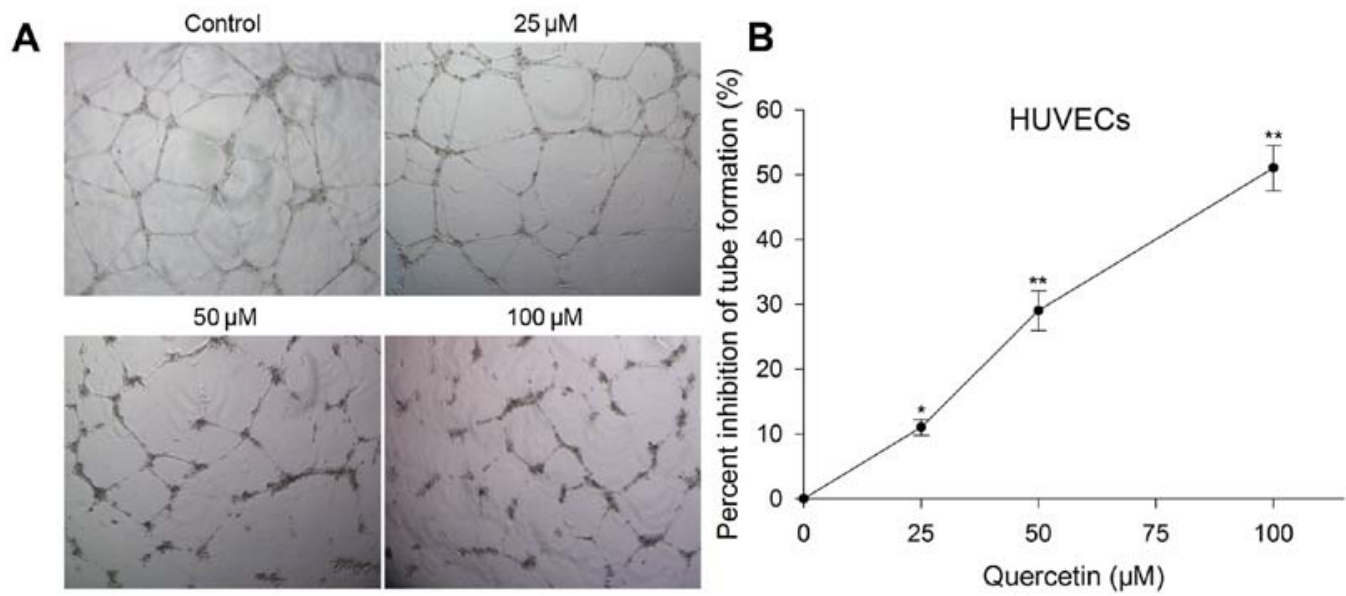

Figure 4. Quercetin inhibited tube formation of HUVECs in vitro. (A) Capillary-like tube formation assay was employed to verify the inhibitory effect of quercetin on HUVECs tube formation. (B) Percent inhibition rate of tube formation is presented as means $\pm \mathrm{SD}$ (mean in triplicate). "P<0.05 compared to untreated control; ${ }^{* *} \mathrm{P}<0.01$ denotes a significant difference between treatment and the control group.

male BALB/c nude mice were randomly assigned and treated with vehicle or quercetin at the dose of 25,50 and $75 \mathrm{mg} / \mathrm{kg}$. At the end of the intervention procedure lasting 4 weeks, xenografts of the four groups were harvested and it was found that PC-3 tumors were smaller in quercetin $25 \mathrm{mg} / \mathrm{kg}$ treated groups than in the control group. The xenografts become much smaller when the drug dose increased (Fig. 6A). The inhibition rate of 25,50 and $75 \mathrm{mg} / \mathrm{kg}$ groups were $22.85,29.6$ and $37.5 \%$, respectively (Fig. 6B and C). In the entire treatment process, even large dose of $75 \mathrm{mg} / \mathrm{kg}$ was well tolerated by the nude mice. Drug-related toxic reaction or side-effects such as poor mental state, convulsion and hematuria were not observed. Weight loss in the treated groups did not show significant difference from the control group (Fig. 6D).

Quercetin inhibits tumor microvessel density in xenograft tumor tissue. Microvessel density examination was carried out to explore the inhibitory effect of quercetin on angiogenesis in vivo. Immunohistochemistry showed that relative $\mathrm{CD} 31$ - and CD34-positive vessels in quercetin $25 \mathrm{mg} / \mathrm{kg}$ group were 


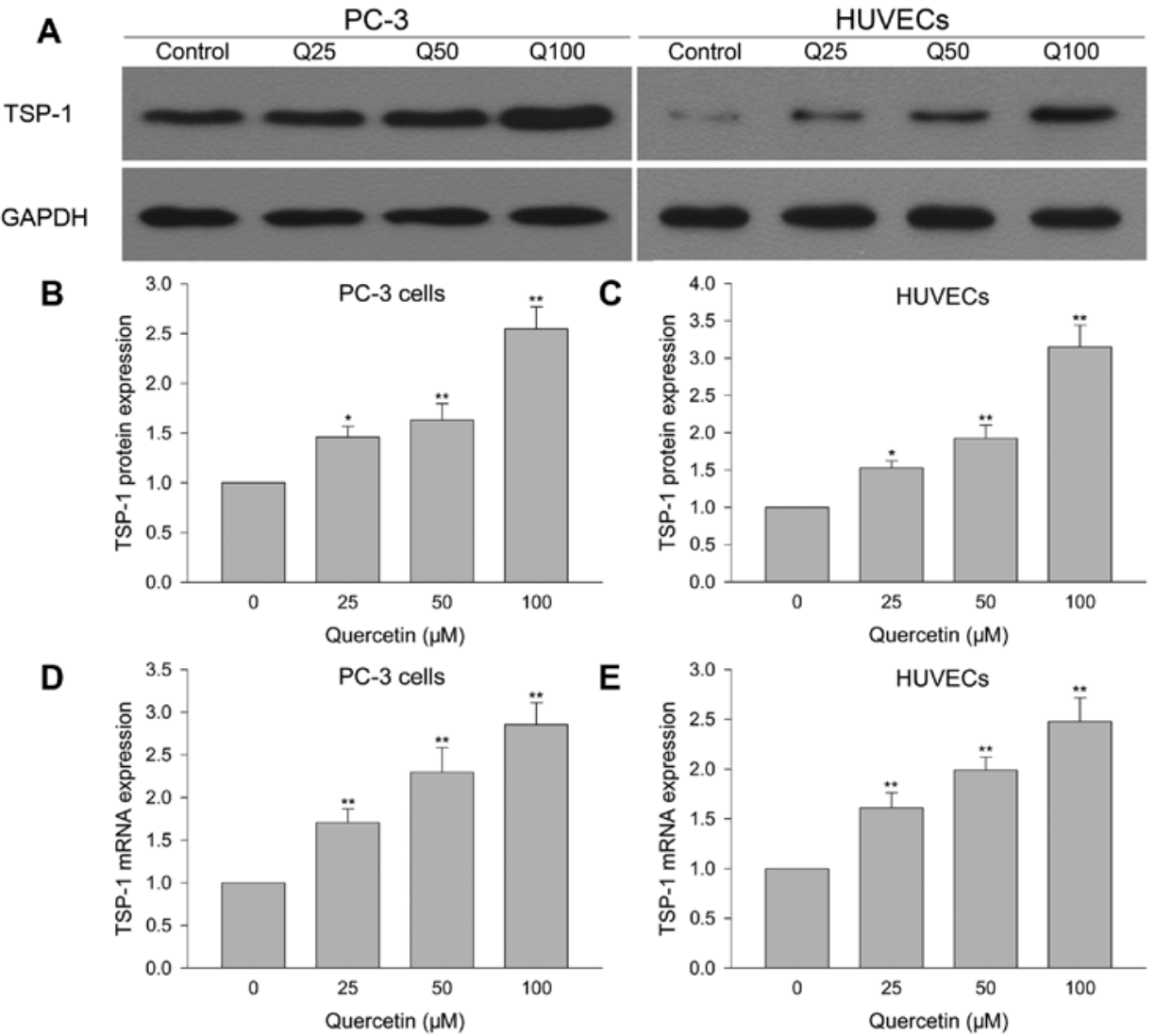

Figure 5. Quercetin decreases TSP-1 protein and mRNA expression in PC-3 cells and HUVECs. (A) TSP-1 protein and (D and E) mRNA expression were examined by western blotting and quantitative real-time PCR. Values of TSP-1 protein (B and C) and mRNA (D and E) are presented as means \pm SD (values from three independent experiments). ${ }^{*} \mathrm{P}<0.05$ compared to untreated control; ${ }^{* *} \mathrm{P}<0.01$ denotes a significant difference between treatment group and the control group.

A

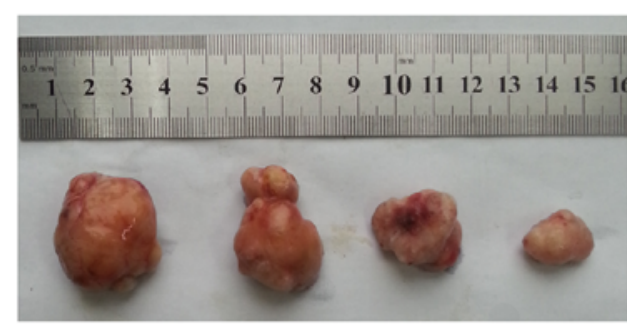

Control $\quad 25 \mathrm{mg} / \mathrm{kg} 50 \mathrm{mg} / \mathrm{kg} 75 \mathrm{mg} / \mathrm{kg}$

C

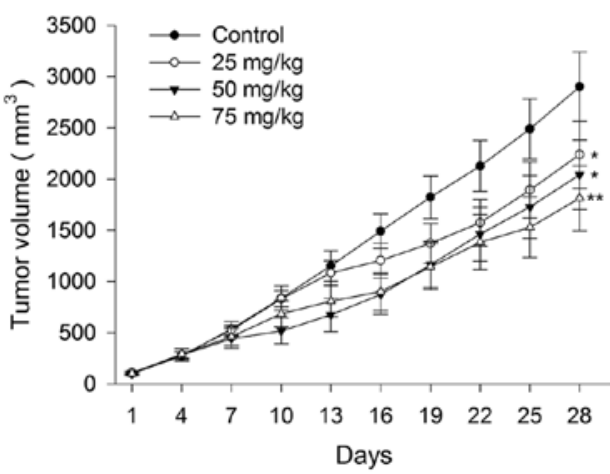

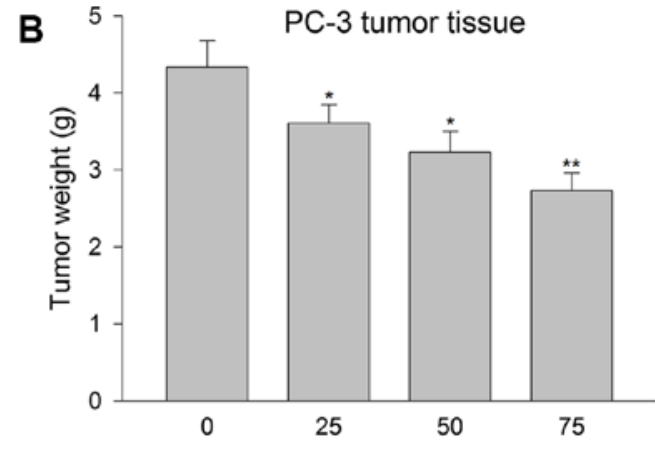

Quercetin $(\mathrm{mg} / \mathrm{kg})$

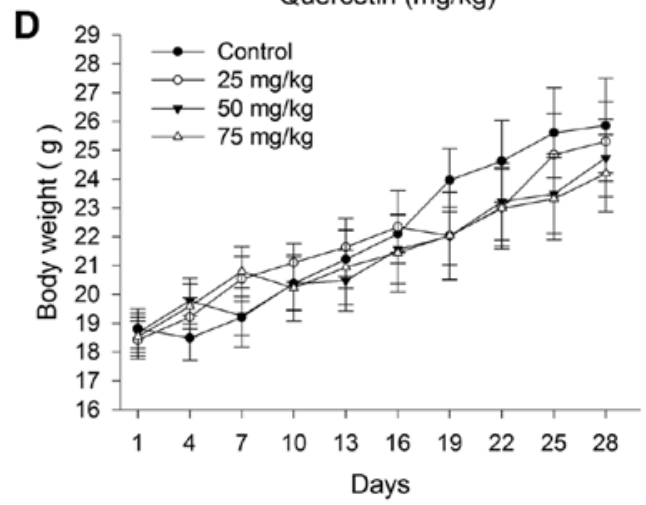

Figure 6. Quercetin inhibits PC-3 xenograft tumor growth. (A) PC-3 xenograft tumors were smaller in quercetin $25 \mathrm{mg} / \mathrm{kg}$ treatment group than the vehicle control, and the inhibition was more remarkable when quercetin was applied at a larger dose. (B) Tumor weight and (C) tumor volume were significantly reduced by quercetin in a dose-dependent manner. There was no significant difference in mouse weight between the groups (D). Data are represented as means \pm SD. ${ }^{*} \mathrm{P}<0.05$ compared with control; ${ }^{* *} \mathrm{P}<0.01$ denotes a significant difference between treatment and the control group. 

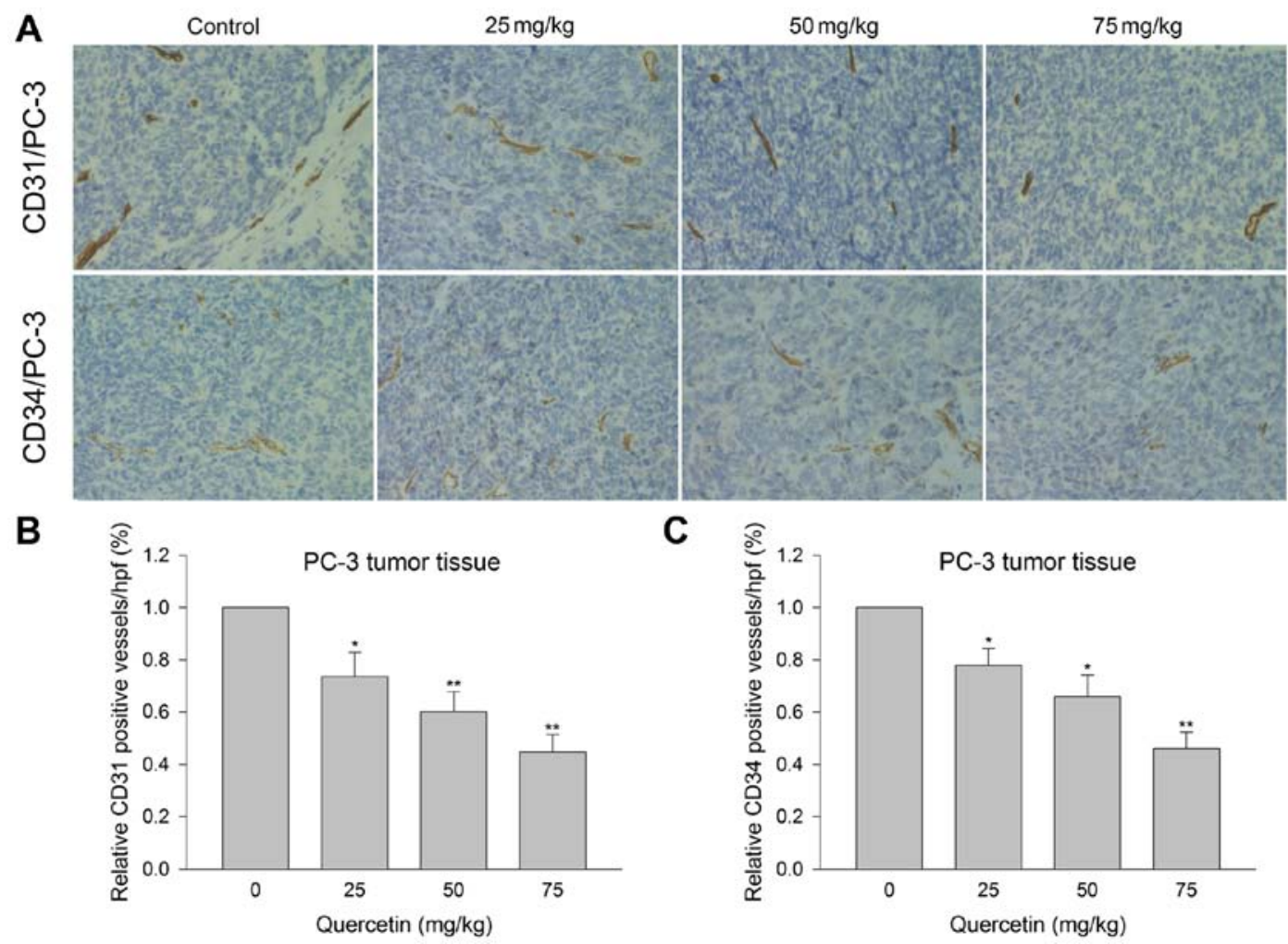

Figure 7. Quercetin decreases microvessel density in PC-3 xenograft tumor tissues. (A) Immunohistochemistry detected CD31- and CD34-positive vessels in PC-3 xenograft tumor tissue. Relative CD31 (B) and CD34 (C) positive vessels are presented as means \pm SD, and expression rate was from three random high powered fields per slide (light microscopy, hpf; magnification, $\mathrm{x} 400$ ). ${ }^{*} \mathrm{P}<0.05$ compared to control; ${ }^{* *} \mathrm{P}<0.01$ denotes a significant difference between treatment and the control group.

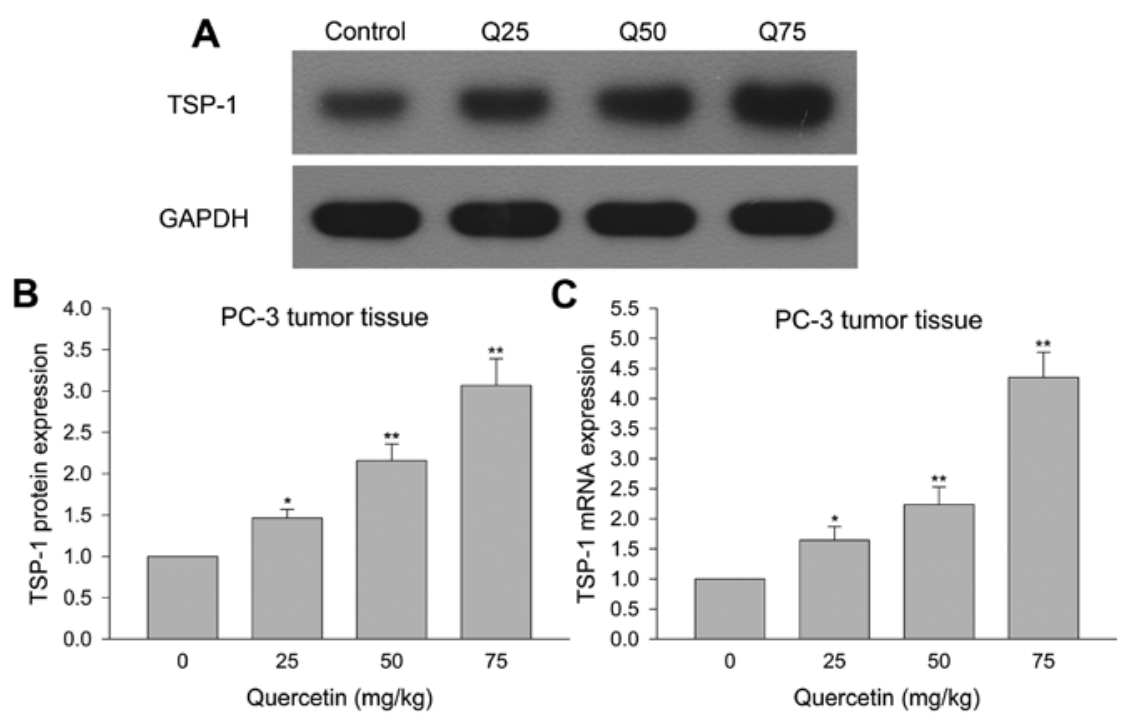

Figure 8. Quercetin reduces TSP-1 protein and mRNA in PC-3 xenograft tumor tissues. (A) TSP-1 protein and (C) mRNA expression were examined by western blotting and quantitative real-time PCR. Values of TSP-1 protein (B) and mRNA (C) are presented as means \pm SD (values from three independent experiments). ${ }^{*} \mathrm{P}<0.05$ compared to untreated control; ${ }^{* *} \mathrm{P}<0.01$ denotes a significant difference between treatment and the control group.

73.5 and $77.8 \%$, respectively, as compared to $100 \%$ in the vehicle control group. The positive rates for CD31 and CD34 were 60.08 and $65.87 \%$, respectively, in quercetin $50 \mathrm{mg} / \mathrm{kg}$ group, and 44.68 and $46.11 \%$, respectively, in $75 \mathrm{mg} / \mathrm{kg}$ group (Fig. 7). The above verified the ability of quercetin to inhibit angiogenesis in prostate cancer cell xenografts in a dose-dependent manner.
Quercetin inhibits TSP-1 protein and mRNA expression in $P C-3$ cell xenograft tumor tissue. Western blotting and Q-PCR detected TSP-1 protein and mRNA expression in xenograft tumor tissues to investigate the reason for quercetin inhibition of prostate cancer PC-3 cell growth in vivo. Fig. 8, shows that quercetin $25 \mathrm{mg} / \mathrm{kg}$ led to increased TSP-1 protein and mRNA 
expression in tumor tissues compared with control, whereas larger doses resulted in greater amount of TSP-1 protein and mRNA. These results indicated that quercetin increased TSP-1 protein and mRNA expression to inhibit angiogenesis thereby leading to antagonize human prostate cancer growth in vivo.

\section{Discussion}

The incidence and mortality rate of prostate cancer have been increasing in recent years, but effective treatments for prostate cancer are scarce and the present comprehensive therapeutic measures cannot achieve satisfactory results $(1,4)$. Based on these disadvantageous situations, researchers are investigating naturally-occurring substances to solve this thorny problem.

Quercetin has presented its promising anticancer property in prostate cancer (7). In vitro, quercetin inhibited prostate cancer cell growth through reducing androgen receptor (AR) and its inducible genes expression (6), proapoptotic and antiproliferative activities (11), and many other possible mechanisms (7). In vivo, quercetin was able to antagonize prostate cancer cell xenograft growth to a large degree by targeting various signal pathways (7). In the present study, it was proven again that quercetin effectively inhibited androgen-independent PC-3 human prostate cancer cell growth to a large degree both in vitro and in vivo, and this anti-prostate cancer action was manifested in a dose- and time-dependent manner. In addition, quercetin is non-toxic. It was well tolerated by experimental animals and did not show any genotoxicity $(8,12,13,17)$. Recently, two clinical studies have been carried out for quercetin and verified that $1,000 \mathrm{mg}$ of quercetin was well tolerated by healthy adults or patients with III chronic prostatitis and this dosage did not produce any side-effects or drug-related toxicity $(18,19)$. Furthermore, quercetin is abundant in various fruits and vegetables and can be sufficiently gained through diet. Therefore, quercetin is safe and effective and has broad application prospects in prostate cancer treatment.

Angiogenesis refers to the process of neovascularization from the existing vascular system and plays an important role in the tumor growth as new blood vessels can provide sufficient oxygen and nutrients. At the same time, tumors themselves can promote more neovascularization to further facilitate tumor development and progression (7). In the absence of local new blood vessel formation, tumors will not grow larger than 2-3 $\mathrm{mm}$ in diameter and its metastasis will be hindered as well (20). Thus tumors can be effectively treated by inhibiting angiogenesis. Moreover, natural inhibitors of angiogenesis are low toxic and will not harm the pre-existing vasculature (20). The above indicated that novel cancer therapies targeting angiogenesis have great therapeutic potential and have attracted attention long ago (21). Our present experiment manifested that quercetin effectively inhibited proliferation, migration, invasion and tube formation of HUVECs and thus confirmed the ability of quercetin to inhibit angiogenesis in vitro. When PC-3 cells were exposed to various doses of quercetin, the proliferation was inhibited significantly in a dose- and timedependent manner. It is speculated that quercetin inhibited prostate cancer cell growth through angiogenesis inhibition and this was further confirmed in vivo. When prostate cancer xenograft tumor bearing nude mice were treated with quercetin, the volume and weight of xenograft tumors were greatly reduced. Immunohistochemical examination showed that microvascular density represented by CD31- and CD34positive vessels was decreased in drug-treated groups and it was more obvious when dose increased. The inhibitory effect of quercetin on angiogenesis in vivo directly resulted in the inhibition of xenograft tumor growth.

Angiogenesis involves a series of processes and procedures that are initiated by angiogenic factors with subsequent relevant signal pathways activated. Vascular endothelial growth factor (VEGF) and hypoxia inducible factor-1 $\alpha$ (HIF-1 $\alpha)$ are the two important angiogenic factors to regulate angiogenesis and have been deemed as the therapeutic target for many anticancer drugs (9,22-24). Thrombospondin-1 (TSP-1) is first isolated from platelet $\alpha$-granules and acts as the endogenous antiangiogenic factor (24). Various substances can achieve the inhibitory effect on angiogenesis and tumor growth through upregulating TSP-1 (5). The possible mechanisms are as follows: direct effects on endothelial cell migration, induction of apoptosis, inhibition of matrix metalloproteinase- 9 activation, and interaction of TSP-1 with CD36 $(20,25)$. Although the present study, and Pratheeshkumar et al verified that quercetin could inhibit prostate cancer growth through antagonizing angiogenesis both in vitro and in vivo $(8,23)$, the important role of TSP-1 upregulation in the angiogenesis inhibition and anti-prostate cancer effect of quercetin has not been previously reported.

We gave different doses of quercetin or DMSO to HUVECs and then cultured for 24, 48 and $72 \mathrm{~h}$. Migration, invasion and tube formation were greatly inhibited. Western blotting and Q-PCR showed that TSP-1 protein and mRNA increased with the dose increase. It is strongly suggested that quercetin inhibited angiogenesis through TSP-1 upregulation. Then PC-3 cells were treated with quercetin, the proliferation was inhibited and TSP-1 protein and mRNA were increased in a dose-dependent manner. This showed that quercetin effectively antagonized prostate cancer cells growth by means of increasing TSP-1 expression. It was already previously clear that quercetin could inhibit human prostate cancer cell growth through angiogenesis inhibition that was mediated via raising TSP-1 protein and mRNA level. However, the quercetin effects on prostate cancer in vivo and the mechanisms involved are not known.

We performed prostate cancer-bearing nude mouse model experiments to explore this issue. When xenografts reached $\sim 100 \mathrm{~mm}^{3}$, mice were randomly assigned and intervened for four weeks. After relevant biochemical examinations were carried out, it was found that both TSP-1 protein and mRNA in the tumor tissues were increased, and the level change had a direct proportional relationship with the drug dose. Microvascular density examination demonstrated that quercetin significantly reduced angiogenesis of xenograft tumor tissues. Large dose such as $75 \mathrm{mg} / \mathrm{kg}$ gave a much stronger inhibitory effect. Therefore, our in vivo results further verified the findings of in vitro experiments that quercetin greatly inhibited angiogenesis by TSP-1 upregulation through which prostate cancer growth was effectively inhibited. However, we did not conducted a deeper and more detailed study on the concrete signal pathway of quercetin inhibiting angiogenesis that mainly involved TSP-1 as well as its upstream and down- 
stream regulated factors. The present study mainly focuses on the effect of quercetin and TSP-1 on angiogenesis and prostate cancer growth both in vitro and in vivo, but mechanism study is still required.

In conclusion, quercetin can effectively inhibit angiogenesis through TSP-1 upregulation to antagonize human prostate cancer PC-3 cell growth in vitro and in vivo. However, the detailed signal pathway concentrating on TSP-1 needs further investigation. The present study can provide a good foundation for the future mechanism research and applying quercetin to clinical study, and it also raise the possibility of using quercetin to treat and prevent human prostate cancer in the near future.

\section{Acknowledgements}

The present study was supported by the National Natural Science Foundation of China (no. 81450027).

\section{References}

1. Siegel RL, Miller KD and Jemal A: Cancer statistics, 2015. CA Cancer J Clin 65: 5-29, 2015

2. Fleshner N: Defining high-risk prostate cancer: Current status. Can J Urol 12 (Suppl 1): S14-S17; discussion 94-96, 2005.

3. Hotte SJ and Saad F: Current management of castrate-resistant prostate cancer. Curr Oncol 17 (Suppl 2): S72-S79, 2010.

4. Qu S, Wang K, Xue H, Wang Y, Wu R, Liu C, Gao AC, Gout PW, Collins CC and Wang Y: Enhanced anticancer activity of a combination of docetaxel and Aneustat (OMN54) in a patientderived, advanced prostate cancer tissue xenograft model. Mol Oncol 8: 311-322, 2014.

5. Olsson A, Björk A, Vallon-Christersson J, Isaacs JT and Leanderson T: Tasquinimod (ABR-215050), a quinoline-3-carboxamide anti-angiogenic agent, modulates the expression of thrombospondin-1 in human prostate tumors. Mol Cancer 9: 107, 2010.

6. Xing N, Chen Y, Mitchell SH and Young CY: Quercetin inhibits the expression and function of the androgen receptor in $\mathrm{LNCaP}$ prostate cancer cells. Carcinogenesis 22: 409-414, 2001.

7. Yang F, Song L, Wang H, Wang J, Xu Z and Xing N: Quercetin in prostate cancer: Chemotherapeutic and chemopreventive effects, mechanisms and clinical application potential (Review). Oncol Rep 33: 2659-2668, 2015.

8. Yang F, Song L, Wang H, Wang J, Xu Z and Xing N: Combination of quercetin and 2-methoxyestradiol enhances inhibition of human prostate cancer LNCaP and PC-3 cells xenograft tumor growth. PLoS One 10: e0128277, 2015.

9. Shi X, Deepak V, Wang L, Ba X, Komori T, Zeng X and Liu W: Thrombospondin-1 is a putative target gene of Runx 2 and Runx 3 . Int J Mol Sci 14: 14321-14332, 2013.

10. Chen Y, Li XX, Xing NZ and Cao XG: Quercetin inhibits choroidal and retinal angiogenesis in vitro. Graefes Arch Clin Exp Ophthalmol 246: 373-378, 2008.
11. Wang G, Song L, Wang $\mathrm{H}$ and Xing N: Quercetin synergizes with 2-methoxyestradiol inhibiting cell growth and inducing apoptosis in human prostate cancer cells. Oncol Rep 30: 357-363, 2013.

12. Sharmila G, Bhat FA, Arunkumar R, Elumalai P, Raja Singh P, Senthilkumar K and Arunakaran J: Chemopreventive effect of quercetin, a natural dietary flavonoid on prostate cancer in in vivo model. Clin Nutr 33: 718-726, 2014.

13. Reiner T, de las Pozas A, Gomez LA and Perez-Stable C: Low dose combinations of 2-methoxyestradiol and docetaxel block prostate cancer cells in mitosis and increase apoptosis. Cancer Lett 276: 21-31, 2009.

14. Ma ZS, Huynh TH, Ng CP, Do PT, Nguyen TH and Huynh H: Reduction of CWR22 prostate tumor xenograft growth by combined tamoxifen-quercetin treatment is associated with inhibition of angiogenesis and cellular proliferation. Int J Oncol 24: 1297-1304, 2004.

15. Ma Z, Hung Nguyen T, Hoa Huynh T, Tien Do P and Huynh H: Reduction of rat prostate weight by combined quercetinfinasteride treatment is associated with cell cycle deregulation. J Endocrinol 181: 493-507, 2004.

16. Borghetti GS, Lula IS, Sinisterra RD and Bassani VL: Quercetin/ beta-cyclodextrin solid complexes prepared in aqueous solution followed by spray-drying or by physical mixture. AAPS PharmSciTech 10: 235-242, 2009.

17. Utesch D, Feige K, Dasenbrock J, Broschard TH, Harwood M, Danielewska-Nikiel B and Lines TC: Evaluation of the potential in vivo genotoxicity of quercetin. Mutat Res 654: 38-44, 2008.

18. Cialdella-Kam L, Nieman DC, Sha W, Meaney MP, Knab AM and Shanely RA: Dose-response to 3 months of quercetincontaining supplements on metabolite and quercetin conjugate profile in adults. Br J Nutr 109: 1923-1933, 2013.

19. Shoskes DA, Zeitlin SI, Shahed A and Rajfer J: Quercetin in men with category III chronic prostatitis: A preliminary prospective, double-blind, placebo-controlled trial. Urology 54: 960-963, 1999.

20. Ren B, Yee KO, Lawler J and Khosravi-Far R: Regulation of tumor angiogenesis by thrombospondin-1. Biochim Biophys Acta 1765: 178-188, 2006.

21. Hanahan D and Folkman J: Patterns and emerging mechanisms of the angiogenic switch during tumorigenesis. Cell 86: 353-364, 1996.

22. Lee DH and Lee YJ: Quercetin suppresses hypoxia-induced accumulation of hypoxia-inducible factor-1alpha (HIF-1alpha) through inhibiting protein synthesis. J Cell Biochem 105: 546-553, 2008

23. Pratheeshkumar P, Budhraja A, Son YO, Wang X, Zhang Z, Ding S, Wang L, Hitron A, Lee JC, Xu M, et al: Quercetin inhibits angiogenesis mediated human prostate tumor growth by targeting VEGFR-2 regulated AKT/mTOR/P70S6K signaling pathways. PLoS One 7: e47516, 2012.

24. Miyata Y and Sakai H: Thrombospondin-1 in urological cancer: Pathological role, clinical significance, and therapeutic prospects. Int J Mol Sci 14: 12249-12272, 2013.

25. Kazerounian S, Yee KO and Lawler J: Thrombospondins in cancer. Cell Mol Life Sci 65: 700-712, 2008. 